\title{
Deciphering Quasar Millilensing - How to Distinguish Between Dark and Luminous Lenses
}

\author{
Heidi Lietzen, Chris Flynn, Erik Zackrisson and Pasi Nurmi \\ Tuorla Observtory, University of Turku, Väisäläntie 20, FI-21500 Piikkiö, Finland \\ email: heilie@utu.fi
}

\begin{abstract}
Dwarf-galaxy sized dark matter halos may be detectable when a bright background source is gravitationally lensed on scales of milliarcseconds. Such effects can be caused by dark galaxies as well as luminous ones. Here we discuss conditions for detecting a luminous lensing object through direct imaging, via measurements of the point spread function (PSF) to subtract the quasar from the image, revealing the quasar host galaxy and any luminous lens. The technique applies to both types of millilensing effect, i.e. magnification and image splitting. We show that luminous dwarf-galaxy lenses should be detectable with this method up to redshifts $z \sim 0.2$.
\end{abstract}

Keywords. gravitational lensing, galaxies: dwarf, quasars: general

\section{Introduction}

The millilensing phenomenon occurs when a small galaxy or dark halo bends the light of a background quasar, forming strongly lensed images on milliarcsecond scales. The observable consequences are magnification and, possibly, milliarcsecond-scale image splittings. The latter effect has not yet been observed, but may be within reach of current radio interferometers.

With the angular resolution characteristic of optical imaging, quasars appear point-like while their host galaxies may be extended sources, which can be revealed by subtracting the central point source, using a PSF based on stars in the image. The furthest host galaxies detected to date with this method are at redshift $z \sim 3$ (Falomo et al. 2006).

In a millilensing system this method could be used for detecting the lensing galaxy, provided that it has a sufficiently high luminosity. Non-detections may furthermore be converted into lower limits on the mass-to-light ratio of the lens. If both the quasar host and the lensing galaxy are seen, it may be necessary to subtract the host galaxy in order to analyse the lens parameters, (although this may be difficult in a lensing system where the host galaxy brightness profile is distorted because of the lensing). Thus an interesting case would be one in which the lensing galaxy is seen, but the quasar host is not. Fortunately, it may be possible to preselect quasars so that this condition is likely to be fulfilled.

\section{Results}

The host and/or lensing galaxies can be detected only if their angular diameter is larger than the seeing disk, so they appear as extended sources. We here take a typical host and a typical dwarf galaxy and estimate their angular radii as a function of redshift. The host is a giant elliptical, 0230-027 (Dunlop et al. 2003) and the dwarf galaxy is N29, a member of the NGC 5044 group (Cellone \& Buzzoni 2001). 


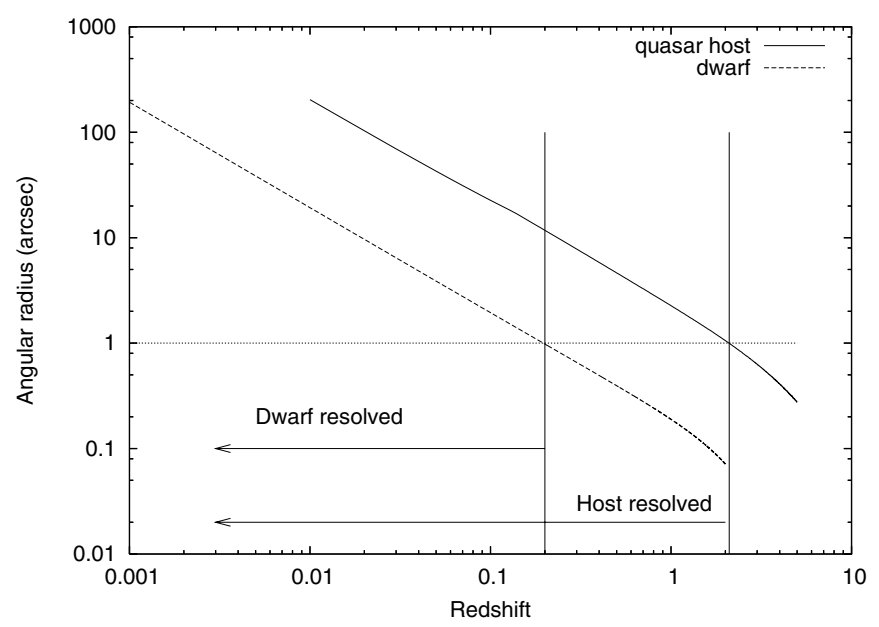

Figure 1. Angular size of the quasar host galaxy and a dwarf galaxy at different redshifts.

The horizontal line shows a typical seeing of a ground based telescope.

For the detection limit we use the Holmberg radius, i.e. the radius at which the surface magnitude is $26.5 \mathrm{mag} \operatorname{arcsec}^{-2}$. To calculate this radius at different redshifts we used observed brightness profiles and applied dimming proportional to $(1+z)^{4}$. The cosmology we used is the $\Lambda$ CDM model with $H_{0}=71 \mathrm{~km} \mathrm{~s}^{-1} \mathrm{Mpc}^{-1}, \Omega_{\Lambda}=0.73$ and $\Omega_{m}=0.27$.

The surface brightness profile of $0230-027$ comes from Dunlop et al. 2003. It is at redshift $z=0.239$ and the Holmberg radius is $\sim 10$ arcseconds. This corresponds to 40 $\mathrm{kpc}$ in physical units. The surface brightness profile for N29 is from Cellone \& Buzzoni 2001 , its Holmberg radius of this galaxy is $\sim 36$ arcsec and we adopt a distance of 23 Mpc which leads to a radius of $4 \mathrm{kpc}$.

Figure 1 shows that using a ground based telescope, the quasar host galaxy can be seen as extended sources out to $z \sim 2$, while the foreground lensing dwarf can be resolved out to redshift $z \sim 0.2$. We did not take the color change or galaxy evolution into account, and these issues could make the galaxies smaller at high redshifts.

These results show that it is possible to have a situation where the lensing galaxy is resolved, but the quasar host galaxy is not. For example, if the redshift of the lens is 0.1 and the redshift of the quasar is 3.0 we should be able to get a clean detection of the lens with a ground based telescope.

We have a paper in preparation on this and other photometric and spectroscopic techniques for separating luminous lenses from dark ones.

\section{References}

Cellone, S. A. \& Buzzoni, A. 2001, A\&SA, 369, 742

Dunlop, J. S., McLure, R. J., Kukula, M. J., et al. 2003, MNRAS, 340, 1095

Falomo, R., Kotilainen, J., Scarpa, R., et al. 2006, New Astron. Revs, 50, 732 